\title{
Simultaneous mapping of the tricuspid and mitral valve annuli at electrophysiological study
}

\author{
Lloyd M Davis, David A Richards, John B Uther, David L Ross
}

\begin{abstract}
Background-Mapping of the right free wall in patients with accessory pathways is difficult compared with that of the left free wall where the coronary sinus permits stable and accurate location of the electrodes used for endocardial mapping. Furthermore, the sequential roving catheter method is less satisfactory than multiple simultaneous electrode recordings spanning the circumference of the valve annulus. A new method for mapping the tricuspid annulus is described.

Methods-Mapping was performed in nine patients with a suspected right free wall accessory pathway or an atriofascicular connection. The tricuspid annulus was mapped using a specially shaped $1 \mathrm{~cm}$ interelectrode 10 pole catheter positioned in the right atrium immediately above the annulus. The coronary sinus was mapped with a $5 \mathrm{~mm}$ interelectrode 10 pole catheter and a $2 \mathrm{~mm}$ interelectrode 10 pole catheter recorded His bundle activity. Catheter positions were confirmed by multiplane fluoroscopy. Electrograms were digitised and recorded simultaneously using a custom computerised mapping system. The position of the multielectrode catheter around the tricuspid annulus relative to that of the coronary arteries was examined by coronary angiography in three patients.
\end{abstract}

Results-Seven right free wall and two posterior septal accessory pathways, and three atriofascicular connections were detected. Ventricular activation adjacent to both valve annuli was mapped in five patients with pre-excitation. The locations of eight of the nine accessory pathways and the three atriofascicular connections were confirmed at operative mapping. One right free wall accessory pathway in a patient with Ebstein's anomaly was not detected at operative mapping. No additional accessory pathways were found at operative mapping or routine 6 month postoperative electrophysiological study, or during a mean (SD) clinical follow up of 22 (7) months. The tricuspid annulus catheter was located during coronary angiography at a mean (SD) of about $2.5(0.7) \mathrm{cm}$ above and parallel to the right coronary artery in the right atrioventricular groove.

Conclusions-This new catheter technique permits rapid detailed mapping of atrial and ventricular activation around the tricuspid annulus with a resolution of at least $\leqslant \% 1 \mathrm{~cm}$, depending on the number and spacing of electrodes in each catheter. The technique was accurate as judged by mapping at surgery. This method is simple and safe compared with that of others for mapping the right free wall via the right coronary artery. It should facilitate detection and ablation of right free wall accessory pathways and atriofascicular connections.

(Br Heart f 1995;73:377-382)

Keywords: electrophysiology; ablation; accessory pathway

Catheter ablation using radiofrequency energy is the preferred method for curing patients with accessory pathways. ${ }^{12}$ Successful ablation requires accurate localisation of all accessory pathways. Improving the speed and accuracy of mapping to reduce fluoroscopy times and the number of radiofrequency applications is one of the major challenges facing electrophysiologists performing radiofrequency ablation.

Accessory pathways in the left free wall and posterior septum are easily mapped by multielectrode catheters placed in the coronary sinus. ${ }^{3}$ In contrast, mapping of the right free wall is difficult because there is no easily accessible structure apart from the right coronary artery that follows the tricuspid annulus. Currently, there are two methods for mapping the right free wall. One uses a multielectrode, deflectable tip catheter or a bipolar catheter with a Brockenbrough-type stillette to record sequential points around the endocardial aspect of the tricuspid annulus. The second uses a 2 French gauge bipole or four pole catheter to record from multiple sites in the right coronary artery. ${ }^{4-6}$ These techniques are vulnerable to the problems associated with any method of sequential mapping that requires moving catheters to different locations. Multiple pathways may be missed 
because of inaccuracy in interpreting the positions of the recording electrodes relative to previous locations. Mapping non-sustained arrhythmias with a sequentially recorded technique is usually time consuming and sometimes impossible because of difficulty in reinducing tachycardia. Similarly, trauma from repositioning catheters may cause transient loss of pathway function and prevent further mapping. ${ }^{7}$ Furthermore, with the steerable catheter technique there is no stable reference to guide placement of an ablation catheter. Although catheters placed in the right coronary artery can be used for this purpose, this approach carries a risk of coronary artery trauma which is not present with endocardial mapping. ${ }^{56} \mathrm{~A}$ technique for simultaneously recording multiple points around most of the endocardial circumference of the tricuspid annulus should overcome these problems. Such an approach may improve the speed and success of radiofrequency catheter ablation of right free wall pathways.

This study reports a new catheter technique for simultaneous multielectrode endocardial mapping of the tricuspid annulus. The accuracy of the technique was validated by mapping at subsequent surgery as this study preceded the application of catheter ablation at our hospital.

\section{Patients and methods}

\section{PATIENTS}

Nine consecutive patients (mean(SD) (range) 24(17) (2-53) years) with a suspected right free wall accessory pathway or atriofascicular connection undergoing electrophysiological study and who were subsequently mapped at surgery between April 1990 and October 1990 were studied. Ebstein's anomaly was identified on the echocardiograms of three patients and confirmed at surgery. The other six patients had structurally normal hearts. Indications for the electrophysiological study were recurrent palpitations in seven patients and documented Wolff-Parkinson-White syndrome in two. The tricuspid annulus catheter was inserted in an additional three patients undergoing right and left heart catheterisation and coronary angiography for investigation of valvular disease to compare its location with

Figure 1 Two views of the metallic former used for shaping the tricuspid annulus catheter during gas sterilisation at $90^{\circ}$ to each other. Note that the former is shaped so that the electrode catheter follows a clockwise course as viewed from above around the tricuspid annulus. the course of the right coronary artery in the atrioventricular sulcus. The protocol used in this study was approved by the Westmead Hospital Research and Ethics Committee and informed consent obtained in all cases.

\section{CATHETER TECHNIQUE FOR MAPPING}

The right free wall was mapped using a $1 \mathrm{~cm}$ interelectrode 10 pole 6 French gauge USCI catheter. This catheter had been preshaped using a specially designed metal former at the time of sterilisation to facilitate its placement around the endocardial circumference of the tricuspid annulus (fig 1). This involved making a $90^{\circ}$ bend in the proximal portion of the catheter with the former. This angle deflexed to $45-60^{\circ}$ when removed from the former. The circular shape of the terminal $12 \mathrm{~cm}$ of the catheter followed the circumference of the tricuspid annulus. The catheter was inserted from the right femoral vein. Correct positioning of the catheter was facilitated by pointing the tip towards the lateral wall of the right atrium, in the right anterior oblique view, during insertion in the heart. The preshaped catheter then tended to assume the correct position parallel to and about $1-2 \mathrm{~cm}$ above the plane of the tricuspid annulus. Placement of the catheter in the plane of the tricuspid annulus was confirmed by comparing the plane of the catheter with the plane of the coronary sinus catheter, in both left anterior oblique and right anterior oblique views, allowing for the fact that the plane of the tricuspid annulus is approximately $15^{\circ}$ more vertical than the mitral valve in the right anterior oblique projection. ${ }^{8}$ Clockwise rotation positioned the electrodes of the catheter closer to the tricuspid annulus. The left anterior oblique view was used to judge the extent of the circumference of the tricuspid annulus covered by the catheter. A $2 \mathrm{~mm}$ interelectrode 10 pole catheter was positioned across the triangle of Koch after placement of the catheter for endocardial mapping of the tricuspid annulus to record both $\mathrm{His}$ bundle activity and the base of the atrial septum. A 5 $\mathrm{mm}$ interelectrode 10 pole catheter was inserted into the coronary sinus from the left antecubital vein to map the left free wall and the posterior septum. A quadpolar or bipolar catheter was placed at the right ventricular apex for recording and pacing. The positions of the electrode catheters were recorded on videotape and photographic paper in the left anterior oblique and right anterior oblique views. Figure 2 illustrates the usual positions of the electrode catheters during mapping.

All electrodes on all catheters were recorded simultaneously. Signals were recorded in the unipolar mode relative to an indifferent electrode placed in the right iliac vein. Bipolar signals were derived from the unipolar electrograms by digital subtraction of adjacent unipolar signals. The signals were amplified 500-1000 times and filtered with a bandpass of $35-500 \mathrm{~Hz}$. The processed signals were recorded on a Masscomp 54S-02E computer and on paper by a Mingograf ink jet recorder for subsequent analysis. 

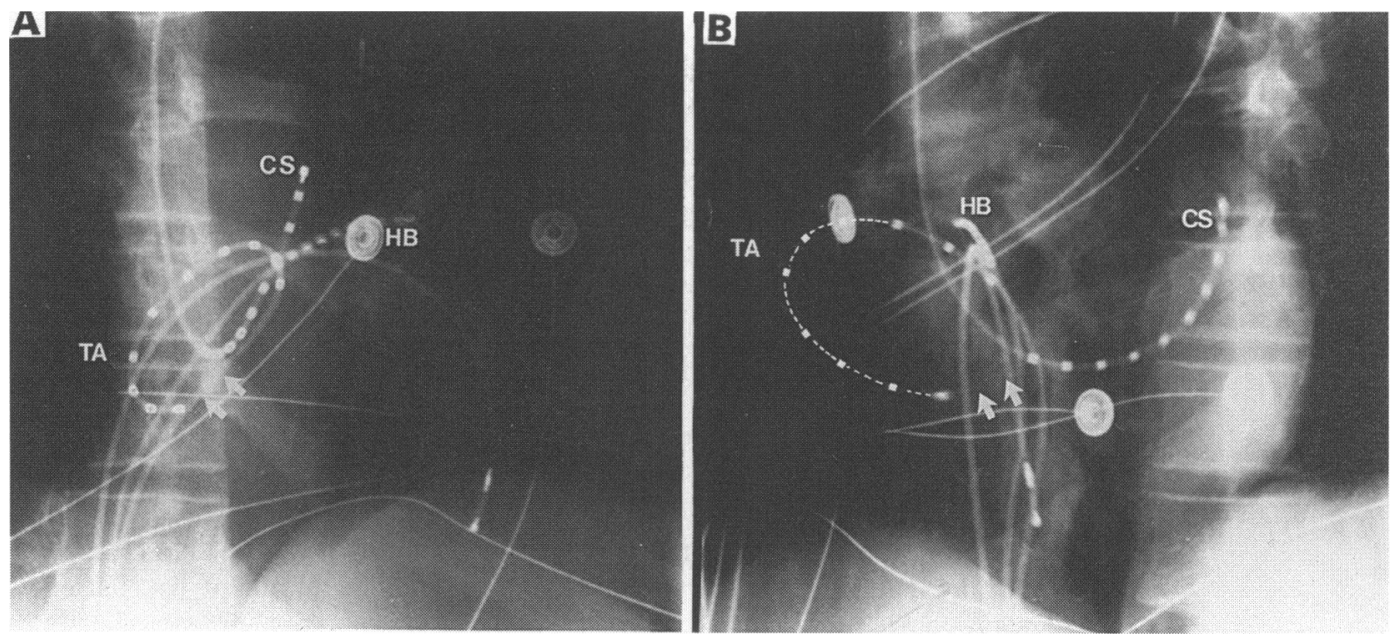

Figure 2 (A) Right anterior oblique view of the usual catheter electrode arrangement used for mapping of accessory pathways. A $5 \mathrm{~mm}$ interelectrode 10 pole catheter has been inserted in the coronary sinus (CS) so that the most proximal electrode is at the coronary sinus orifice. A $10 \mathrm{~mm}$ interelectrode 10 pole catheter (TA) has been positioned around the atrial side of the tricuspid annulus. The plane of this catheter is similar to that of the coronary sinus catheter. A2 mm interelectrode 10 pole catheter $(H B)$ has been placed at the region of the His bundle to record activation over the normal conduction system. A bipolar catheter has been placed at the right ventricular apex for pacing. The catheters encircle most of the relevant portions of the valve annulus. Note that a $2 \mathrm{~mm}$ portion of the tricuspid annulus between the tip of the tricuspid annulus and the coronary sinus orifice is not well covered with this initial version of the tricuspid annulus catheter (arrows). (B) The same catheter arrangement viewed in the left anterior oblique projection.

\section{OPERATIVE MAPPING}

Operative mapping was performed using the same techniques described previously by Gallagher et al. ${ }^{910}$ In brief, a hand held $1 \mathrm{~mm}$ interelectrode bipolar probe was used to map the heart around the atrioventricular groove according to a predetermined grid. The timing of local atrial activation was compared with that of a reference electrode located on the left atrial appendage. Local ventricular activation was measured relative to that of a reference electrode sutured to the anterior wall of the right ventricle.

\section{DEFINITIONS}

Local activation was regarded as the steepest negative slope for unipolar recordings and as the point of greatest signal amplitude above or below the isoelectric line for bipolar recordings.

The atrial insertion sites of accessory pathways were determined with the catheter technique by mapping during orthodromic tachycardia, and sequentially pacing each pair of electrodes around the atrioventricular rings at a cycle length of $400 \mathrm{~ms}$. The earliest site of atrial activation during orthodromic tachycardia and the shortest stimulus to delta time during atrial pacing indicated the atrial insertion site of the accessory pathway or the atriofascicular fibre. Earliest ventricular activity during maximal pre-excitation determined from the catheter electrodes localised the ventricular end of the pathway. Additional accessory pathways were considered to be present if during orthodromic tachycardia another portion of the atria adjacent to the valve annuli was activated at least $4 \mathrm{~ms}$ earlier than all other surrounding atrial sites within $0.5-1.0 \mathrm{~cm}$ of this location. A difference of at least $4 \mathrm{~ms}$ was chosen because this was twice the error in marking electrograms and because this allowed the computer to sample four points from the original electrogram. The location of the atrial end of an atriofascicular fibre was defined as the site at which the shortest stimulus to delta interval was recorded during atrial pacing at a constant cycle length with stable pre-excitation.

The sequence of ventricular activation next to the atrioventricular valve annuli was determined at operative mapping during preexcitation present during sinus rhythm or induced by pacing the left atrial appendage at an appropriate cycle length. The same methods, as for mapping with the catheter technique, were used to locate the atrial insertion sites of the accessory pathways and atriofascicular fibres.

\section{COMPARISONS}

The accuracy of the new catheter technique for endocardial mapping was assessed by comparing the number and locations of the accessory pathways detected with those found by mapping at surgery. In addition, the clinical outcome was assessed by follow up in all patients and at late electrophysiological study in five to determine whether any accessory pathways had recurred, thus indicating a possible error in mapping.

\section{ANATOMICAL LOCATION OF THE CATHETER USED FOR MAPPING}

The location of the multielectrode catheter used for mapping the tricuspid annulus was studied in three separate patients undergoing coronary angiography to determine the relation to the course of the right coronary artery in the right atrioventricular groove. These patients were all undergoing right and left heart catheterisation for investigation of valvular heart disease. The mapping catheter was positioned around the tricuspid annulus 
after conclusion of the right heart study Cineangiograms were then recorded in both the left anterior oblique and right anterior oblique views during contrast injection of the right coronary artery and used to determine the position of the catheter for endocardial mapping relative to that of the right coronary artery.

\section{Results}

CATHETER TECHNIQUE FOR MAPPING OF THE TRICUSPID ANNULUS

The tricuspid annulus catheter was readily positioned and satisfactory electrograms obtained in all patients. Nine accessory pathways and three atriofascicular connections were detected and mapped with this technique. Three patients had two accessory pathways.

Atrial mapping was performed during orthodromic tachycardia in all patients with accessory ventriculoatrial pathways. Maps of the atrial insertion sites were also generated by atrial pacing at sequential sites around the tricuspid annulus in five patients with preexcitation (two with an accessory pathway and three with an atriofascicular fibre). The pathways did not conduct in the anterograde direction in the remaining patients. Stimulus to delta mapping around the tricuspid annulus was the only method for locating the atrial insertion sites of the atriofascicular fibres as these fibres did not conduct in the retrograde direction. A mean (SD) of seven (three) atrial sites around the tricuspid annulus was paced per patient. Reliable atrial capture was not obtained from one of the attempted sites. In this patient, however, easily interpretable atrial electrograms were still recorded from the relevant electrode pair. The atrial insertion sites of the anterograde conducting pathways found by measurement of stimulus to $d$ intervals, during atrial pacing, and by mapping during orthodromic tachycardia were concordant in the two patients with bidirectional pathways.

Ventricular mapping during pre-excitation was performed using the tricuspid annulus catheter in the two patients with WolffParkinson-White syndrome. In one case, the ventricular insertion site of the anterior right free wall accessory pathway was $1 \mathrm{~cm}$ lateral to the atrial insertion site. In the other, the ventricular insertion site was in the same position in the posterior septum as the atrial insertion site of the accessory pathway. Mapping of the sequence of right ventricular activation adjacent to the tricuspid annulus was not relevant in those with an atriofascicular fibre as these fibres insert into the right bundle branch or the distal right ventricle.

CORRELATION WITH OPERATIVE MAPPING

Surgical cure was attempted in all nine patients. Operative mapping confirmed the location of all but one of the accessory pathways. A posterolateral right free wall accessory pathway was not detected at operative mapping in one patient. As this patient had
Ebstein's anomaly and the preoperative catheter map provided convincing evidence of an additional right free wall accessory pathway, the posterolateral right free wall was also dissected.

COMPLICATIONS

Atrial fibrillation or flutter was not precipitated in any patient by insertion and positioning of the tricuspid annulus catheter. Nor were any other complications encountered, such as atrial perforation, during insertion and positioning of the catheter.

\section{LONG-TERM OUTCOME}

None of the patients had an accessory pathway or atriofascicular connection during a mean (SD) (range) follow up of 22(7) (6-28) months. Electrophysiological study was performed via epicardial wires 1 week after surgery in eight patients. One 2 year old patient did not have an electrophysiological study 1 week after surgery but was studied 6 months after operation. A 6 month postoperative electrophysiological study was performed in five patients. All these studies were normal. Four patients who refused to undergo electrophysiological study 6 months after surgery were followed up by telephone interview. None of these patients had symptoms suggesting that supraventricular tachycardia occurred after operation.

\section{ANGIOGRAPHIC DATA}

The position of the catheter for mapping the tricuspid annulus was compared with the location of the right coronary artery in three patients undergoing coronary angiography. The mapping catheter was located parallel to and within a mean (SD) (range) of $2 \cdot 5(0 \cdot 7)$ $(1-3) \mathrm{cm}$ above the right coronary artery and $0-2 \mathrm{~cm}$ above the tricuspid annulus (as the right coronary artery is often $1 \mathrm{~cm}$ below the fibrous valve annulus) in all three patients. ${ }^{11}$ The catheter did not cover a $2 \mathrm{~cm}$ portion of the tricuspid annulus between the coronary sinus orifice and the posterolateral right free wall (fig 2). The tip of the catheter usually became fixed in the crista terminalis or Eustachian valve at the posterolateral margin of the right atrium preventing further advancement of the catheter past this region.

\section{Discussion}

This study demonstrated that the tricuspid annulus can be mapped in detail with a single specially shaped 10 or more pole electrode catheter. Insertion and positioning of this catheter was simple. This technique proved to be an accurate method for locating accessory pathways compared with the results of operative mapping. Combination with a multielectrode catheter in the coronary sinus permitted detailed simultaneous mapping of both atrioventricular valve annuli without the need to move the catheters used for mapping. As a consequence, it should improve detection of all accessory pathways. It should also reduce the time required for mapping and 
therefore total fluoroscopy and procedure times. Only one brief episode of supraventricular tachycardia is needed for detailed mapping. Similarly, maps of ventricular activation during pre-excitation are generated more quickly with this technique than with a sequential roving electrode method.

\section{IMPLICATIONS FOR RADIOFREQUENCY \\ ABLATION}

Catheter ablation of right free wall accessory pathway and atriofascicular fibres requires precise localisation of the accessory connection. Although pathways and atriofascicular connections can be located using only a roving deflectable ablation catheter for mapping, this is not always easy. Sequential roving electrode mapping is prone to missing multiple accessory pathways, particularly if orthodromic tachycardia is non-sustained or frequently terminated by atrial extrasystoles, or if there is temporary abolition of pathway function due to catheter induced trauma preventing mapping during ventricular pacing.? Pathways may also be missed if there is inaccurate interpretation of the position of the ablation catheter and therefore the anatomical origin of the electrograms used for generating a sequential map of activation around the tricuspid annulus. In addition, atrial pace mapping is required to identify the atrial insertion sites of atriofascicular connections. This can be technically difficult with a roving octapolar or ablation catheter. ${ }^{12}$ These problems may explain the slightly higher early recurrence rate of right free wall accessory pathways compared with that of left free wall accessory pathways after catheter ablation. ${ }^{13}$ In contrast, simultaneous maps of the tricuspid annulus recorded by the multielectrode catheter provide the electrophysiologist with reliable data on the location of all accessory pathways in the right free wall even when tachycardia is non-sustained and eliminate errors in identifying pathways associated with incorrect interpretation of the site of the recording electrode. In addition, atrial pace maps can be easily generated with the new multielectrode catheter to help locate atrial insertions of atriofascicular fibres and ventricular insertions of right sided accessory pathways. Because of these considerations, rapid mapping provided by the multielectrode catheter may shorten times currently spent using the roving electrode catheter to regionalise the location of accessory pathways before ablation and reduce time spent after ablation confirming that all accessory pathways have been destroyed. Furthermore, initial detailed mapping of the tricuspid annulus with the multielectrode catheter restricts the area needed to be mapped in fine detail with the ablation catheter to a small about $1-2 \mathrm{~cm}$ region marked by the relevant electrodes on the tricuspid annulus catheter. Multielectrode mapping along the atrioventricular valve annuli may also provide data on the direction of the accessory pathway across the atrioventricular sulcus, ${ }^{3}$ which may prove helpful during final positioning of an ablation catheter.
These ideas reflect current practice for ablation of left free wall accessory pathways. Most electrophysiologists use a multielectrode catheter in the coronary sinus to localise the area of interest and focus subsequent mapping with the ablation catheter. Even advocates of a single electrode catheter technique for ablation of pathways in the left free wall recommend use of a multielectrode catheter in the coronary sinus for difficult cases. ${ }^{14}$ To date it has not been possible to use a similar approach for ablating pathways in the right free wall because of the absence of a suitable multielectrode catheter to map the tricuspid annulus. Some electrophysiologists have developed a small bipolar electrode catheter that is positioned in the right coronary artery to map the right free wall and to guide positioning of an ablation catheter. ${ }^{5615}$ This approach, however, exposes the patient to additional risks of coronary artery trauma and arterial cannulation and does not allow the entire right free wall to be mapped simultaneously. Our study reports an alternative simple, low risk transvenous catheter technique to rapidly map the right free wall and guide placement of an ablation catheter. We now routinely use this tricuspid annulus catheter for all electrophysiological studies and radiofrequency ablation involving either right free wall accessory pathways or atriofascicular connections. McClelland et al ${ }^{12}$ have also found a modified version of our catheter to be helpful for locating atrial insertions of atriofascicular connections during radiofrequency ablation in a series of 11 patients.

\section{IS THE CATHETER POSITION OPTIMAL?}

The tricuspid annulus catheter lies in a good position parallel to but a mean (SD) of $2 \cdot 5(0 \cdot 7) \mathrm{cm}$ above the tricuspid annulus. This value may be an overestimate because the right coronary artery, which was used as a marker of the tricuspid annulus, can be located up to $1 \mathrm{~cm}$ below the annulus. ${ }^{11}$ The failure of the catheter to lie exactly on the tricuspid annulus in our study did not lead to any clinically significant errors but in theory could limit the resolution for detecting closely adjacent multiple pathways.

\section{FUTURE IMPROVEMENTS}

Currently only 10-12 electrodes are available on catheters used for endocardial mapping. The tricuspid annulus, however, has an average circumference of $11-13 \mathrm{~cm} .{ }^{16}$ Improvements in catheter design that allow 15-25 or more electrodes to be placed at 5 $\mathrm{mm}$ interelectrode intervals over the distal end of the catheter would provide better mapping resolution. If even more electrodes could be placed on the catheter close bipolar pairs could be used at each mapping site to facilitate detection of accessory pathway potentials.

The catheter did not cover a $1-2 \mathrm{~cm}$ segment in the posterior right free wall because the tip became caught on the crista terminalis or the Eustachian valve. This problem could be overcome by insertion of a pull wire to 
deflect the catheter tip, thus enabling the full circumference of the tricuspid annulus to be mapped with one catheter. Alternatives include positioning the tip of the catheter in the coronary sinus orifice and advancing the remainder of the catheter around the tricuspid annulus, and inserting another multielectrode catheter with only the tip in the coronary sinus so that more proximal electrodes cover the posterior portion of the tricuspid annulus. Introduction of a second pull wire to adjust the plane of tilt of the ring portion of the catheter relative to the axis of the catheter shaft, so that it lies closer to the valve annulus, may also enhance the accuracy and resolution of mapping, particularly if the catheter incorporates a larger number of electrodes.

\section{LIMITATIONS}

Variation in atrial size may necessitate several sizes of curves on the end of the catheter for endocardial mapping to fit each tricuspid annulus. Incorporation of a pull wire to adjust the diameter of the circular tip of the catheter should solve this problem.

Although the catheter is usually stable in position, it may be displaced by an ablation catheter. This rarely occurs, however, and repositioning of the catheter is relatively easy.

Positioning of the tricuspid annulus catheter is associated with a small risk of precipitating atrial fibrillation and other cardiac arrhythmia, and of causing atrial perforation. These problems did not occur in the patients studied but can be expected to occur rarely during insertion of any type of catheter into the right atrium.

\section{Conclusions}

This new technique permits detailed mapping of the tricuspid annulus using 10 or more simultaneously recorded electrodes. It is safe and simple to perform. The findings were accurate as judged by the results of operative mapping. Use of this technique should facilitate rapid detection and localisation of single and multiple right free wall accessory pathways and should expedite catheter ablation or surgery for accessory pathways and atriofascicular fibres.

We thank Peter Caval for his help in constructing equipment, and Vicki Eipper, the technicians, and nursing staff of the electrophysiology laboratory for their help in performing the electrophysiology laboratory for their help in performing the mapping studies. This work was supported by a National
Heart Foundation grant no PM 100 and grant in aid no G $90 S 3026$ (National Heart Foundation of Australia, PO Box 2 Woden, ACT, 2606).

1 Jackman WM, Wang XZ, Friday KJ, Roman CA, Moulton KP, Beckman KJ, et al. Catheter ablation of accessory atrioventricular pathways (Wolff-ParkinsonWhite syndrome) by radiofrequency current. $N$ Engl $\mathcal{F}$ Med 1991;324:1605-11.

2 Kuck KH, Schluter M, Geiger M, Siebels J, Duckeck W. Radiofrequency current catheter ablation of accessory Radiofrequency current catheter ablation of accessory
atrioventricular pathways. Lancet 1991;337:1557-61.

3 Jackman W, Friday KJ, Yeung LWJ, Fitzgerald DM, Beck $\mathrm{B}$, Bowman AJ, et al. New catheter technique for
ackman W recording left free wall accessory atrioventricular pathway activation: identification of pathway fiber orientation. Circulation 1988;78:598-611.

4 Gallagher JJ, Sealy WC, Kasell J, Wallace AG. Multiple accessory pathways in patients with the pre-excitation syndrome. Circulation 1976;54:571-91.

5 Swartz JF, Cohen AI, Fletcher RD, Jones JL, Weston LT Right coronary epicardial mapping improves accessory pathway catheter ablation success [abstract]. Circulation 1989;80(suppl II):431.

6 Lesh M, Van Hare GF, Chien WW, Scheinman MM Mapping in the right coronary artery as an aid to radiofrequency ablation of right sided accessory pathways [abstract]. PACE 1991;14:671.

7 Novick TL, Pritchett EL, Campbell RW, Rogers GC Wallace AG, Gallagher JJ. Temporary, catheter-induced Wallace AG, Gallagher J. Temporary, catheter-induced
block in accessory pathways. Circulation 1978;58: 932-40.

8 McAlpine $\mathrm{W}$. The mitral valve, heart and coronary arteries. Berlin: Springer-Verlag, 1975:55

9 Gallagher JJ, Kasell J, Sealy WC, Pritchett EL, Wallace AG. Epicardial mapping in the Wolff-Parkinson-White syndrome. Circulation 1978;57:854-66.

10 Gallagher J, Gilbert M, Svenson RH, Sealy WC, Kasell J, Wallace AG. Wolff-Parkinson-White syndrome: the problem, evaluation, and surgical correction. Circulation 1975;51:767-85.

11 McAlpine WA. Heart and coronary arteries. Berlin: Springer-Verlag, 1975:106.

12 McClelland JH, Wang X, Beckman KJ, Hazlitt HA, Prior MI, Nakagawa $\mathrm{H}$, et al. Radiofrequency catheter ablation of right atriofascicular (Mahaim) accessory pathtion of right atriofascicular (Mahaim) accessory pathways guided by accessory path

13 Miles WM, Zipes DP, Klein LS. Ablation of free wall accessory pathways. In: Zipes DP, ed. Catheter ablation of arrhythmias. Armonk: Futura Publishing, 1994;1: 224-5.

$14 \mathrm{Kuck} \mathrm{KH}$, Schluter M. Single-catheter approach to radiofrequency current ablation of left-sided accessory pathways in patients with Wolff-Parkinson-White syndrome. Circulation 1991;84:2366-75.

15 Cappato $R$, Schlüter M, Weib C, Siebels J, Hebe J, Duckeck W, Mletzko RU, Kuck KH. Catheter-induced mechanical conduction block of right sided accessory fibers with Mahaim type preexcitation to guide radiofrequency ablation. Circulation 1994;90:282-90.

16 Davies MJ, Pomerance A, Lamb D. Techniques in examination and anatomy of the heart. In: Pomerance A, nation and anatomy of the heart. In: Pomerance A,
Davies MJ, eds. The pathology of the heart. Oxford: Davies MJ, eds. The path
Alden Press, 1975:21-2. 